\title{
OTHONOMTCS
}

Revista de economía, empresa y sociedad

Dosier «E-learning en la economia y la empresa»

ARTÍCULO

\section{La importancia del feedback en un entorno virtual de aprendizaje}

\section{Josep-Maria Batalla-Busquets \\ Dolors Plana Frta \\ María-Jesús Martínez-Argüelles}

Profesores de los Estudios de Economía y Empresa (UOC)

RESUMEN Existe un amplio consenso en que uno de los elementos clave para garantizar un buen proceso de aprendizaje en un contexto universitario no presencial es la existencia de procesos que pauten y dirijan el aprendizaje, y que permitan a los estudiantes evaluar periódicamente su grado de logro de los conocimientos. Una estrategia habitual para facilitar esta pauta es el uso del feeback entre el estudiante y el profesor. Pero en entornos con grupos de estudiantes de gran dimensión, la realización de este feedback puede convertirse en un trabajo demasiado laborioso y no sostenible.

En este artículo presentamos los resultados de tres proyectos piloto centrados en el contexto del grado de Administración y Dirección de empresas (ADE) de la UOC, donde hemos explorado varias herramientas TIC (audio, vídeo y capturas de pantalla) y distintas estrategias para realizar el feedback con el objetivo de hacerlo sostenible en grupos grandes y mejorar la eficacia y eficiencia del proceso de regulación de los aprendizajes.

PALABRAS CLAVE feedback; feedback personalizado; e-feedback; e-learning

\section{The Importance of Feedback in a Virtual Learning Environment}

ABSTRACT There is a high consensus in considering regulatory processes of learning as one of the key elements to ensure the knowledge attainment in distance university context, which allows students to periodically evaluate their learning process. In asynchronous learning environments, one of the normal strategies to facilitate this regulation is the use of feedback mechanisms between teacher and students. Though, in an environment with large groups of students, teacher feedback may become a tedious and unsustaianable task.

In this paper, we present the results of three pilot projects centred in the context of the Business degree from UOC, in which we have explored ICT tools (audio, video and screenshots) and differents stretagies to give feedback with the aim of improving the effectiveness and efficiency of the regulatory process of learning.

KEYWORDS feedback; personalized feedback; e-feedback; e-learning 


\section{Introducción}

El Espacio Europeo de Educación Superior (EEES) ha provocado un cambio en la conceptualización del aprendizaje de los estudiantes en el contexto universitario. Se ha pasado de un proceso basado en la transmisión directa de conocimiento del docente hacia el estudiante a otro, donde el foco está centrado en el proceso de aprendizaje del estudiante. Es decir, el estudiante pasa a ocupar un papel central y activo en la adquisición de conocimiento. Esto está en consonancia con la literatura existente relativa al aprendizaje centrado en el estudiante (Lea et al., 2003).

En el contexto de educación superior en línea, asíncrona y basada en el lenguaje escrito, la información proporcionada por el profesor es un elemento básico en el proceso de aprendizaje de los estudiantes (Higgins et al., 2002; Laurillard, 2002). Es en este contexto que el aprendizaje centrado en el estudiante requiere: (1) promover la capacidad de autoregulación de los estudiantes, (2) aplicar nuevos enfoques de enseñanza y aprendizaje, (3) apoyo efectivo y (4) focalizar las estructuras de orientación de la universidad más claramente en el estudiante. Por lo tanto, la función del apoyo y orientación, junto con una estrecha relación entre el docente y la estudiante, son elementos básicos para garantizar que cada estudiante pueda lograr los objetivos y las competencias propias de la asignatura, así como la capacidad de autoregulación.

Con relación a lo que acabamos de decir, a la hora de obtener buenos resultados de aprendizaje, el factor individual más potente, en comparación con otros aspectos de la docencia, es el feedback. (Black et al., 1998; Hattie, 1987).

\section{1. ¿Qué entendemos por feedback?}

Para dar respuesta a esta cuestión, nos hace falta primero hacer un breve repaso de cuáles son los criterios más comunes para clasificar las diferentes tipologías de feedback. Los criterios que proponemos son: (1) a quién va dirigido el feedback, (2) en qué momento realizamos el feedback, (3) qué contenido se quiere transmitir y (4) qué extensión y formato debe tener.

\section{1. ¿A quién dirigimos el feedback?}

Esta es la primera pregunta que debemos contestar antes de ponernos a elaborar el feedback. En función de quién es o son los destinatarios, podremos ajustar el contenido que queremos trasmitir. Los destinatarios pueden ser grupos o individuos. De este modo, en función del grado de personalización del feedback, podemos distinguir tres categorías:

- Feedback para todo el grupo: Es el menos personalizado, se corresponde a las soluciones enviadas después de cada prueba de evaluación continua (PEC) o a algunos mensajes en el aula, que comentan errores generalizados de las pruebas.

- Feedback a un colectivo. Es un feedback para un colectivo que tiene algo en común, por ejemplo, todos los estudiantes que han obtenido una misma calificación. Muy a menudo sirve para orientar sobre algún tipo de error muy común o para motivar a un colectivo específico.

- Feedback personalizado. De todas las tipologías de retorno, este es el que tiene un mayor impacto en el rendimiento del estudiante

\section{2. ¿En qué momento hacemos feedback?}

Una segunda aproximación para clasificar el feedback es el momento que este retorno se elabora y se transmite. Proporcionar un feedback preventivo, inmediato o diferido ha sido objeto de discusión en numerosas investigaciones sobre esta temática. Inspirados en la categorización de Dempsey et al. (1988) definimos las siguientes categorías: 
- Feedback previo o preventivo. A menudo, antes de que los estudiantes envíen los ejercicios de evaluación continua, aparecen dudas, tanto individuales como colectivas. Este feedback orienta previamente hacia una respuesta correcta.

- Feedback inmediato. Es el que proporciona información al estudiante sobre la respuesta correcta y se corresponde en este entorno a las soluciones genéricas de las PEC, que se publican un día después de la entrega de las soluciones por parte de los estudiantes.

- Feedback diferido. En él se comentan los errores cometidos, se recomiendan contenidos y estrategias para mejorar el proceso de aprendizaje. Muy a menudo es personalizado y da valor añadido a la solución de la PEC.

\section{3. ¿Qué contenidos queremos transmitir?}

En tercer lugar, podemos analizar el feedback en función a los contenidos que transmite. En este sentido, encontramos cuatro tipos de feedback, de menor a mayor grado de complejidad, que son complementarios entre ellos:

- Identificación y corrección de errores: Este feedback informa y corrige los errores cometidos.

- Respuesta correcta: el feedback muestra la respuesta correcta y da la solución adecuada.

- Mejora de tareas: el feedback recomienda contenidos y estrategias para mejorar el trabajo o la prueba que se ha presentado.

- Información en profundidad: el retorno sugiere información en profundidad para seguir avanzando en el aprendizaje en el futuro.

\section{4. ¿Qué extensión y formato debe tener?}

\subsubsection{Extensión}

Otra de las particularidades del feedback en un entorno virtual es su extensión. Esta está estrechamente relacionada con el tipo de conocimiento sobre el cual se da el feedback (según sea más abstracto o conceptual o más aplicado y procedimental). Para que el feedback sea efectivo, tiene que facilitar suficiente información a los estudiantes para que logren los objetivos de aprendizaje previstos.

\subsubsection{Formato}

El feedback puede tomar diferentes formas: texto y/o tecnologías multimedia (grabaciones en audio y en vídeo, captura de pantalla con audio, así como la posibilidad de colgar vídeos comprimidos). El estudiante lo recibe dentro del registro de evaluación continua, bien mediante un fichero adjunto, o bien dentro del cuerpo de un mensaje.

Este feedback, además de contribuir a mejorar el proceso de aprendizaje de los estudiantes, es un instrumento clave de motivación de los mismos, para que no abandonen las asignaturas y sigan el proceso de evaluación continuo.

A continuación, en la tabla 1 se presentan varias modalidades de feedback según el momento en que tiene lugar. 
Tabla 1. Modalidades de feedback

\begin{tabular}{|c|c|c|c|c|}
\hline $\begin{array}{l}\text { Momento del } \\
\text { feedback }\end{array}$ & $\begin{array}{l}\text { Qué contenidos } \\
\text { se transmiten }\end{array}$ & $\begin{array}{l}\text { A quien } \\
\text { lo dirigimos }\end{array}$ & $\begin{array}{l}\text { Actitud del } \\
\text { consultor }\end{array}$ & Formato \\
\hline $\begin{array}{l}\text { Preventivo. Previo a la } \\
\text { entrega de la PEC }\end{array}$ & $\begin{array}{l}\text { Identificación y } \\
\text { corrección de errores }\end{array}$ & $\begin{array}{l}\text { Aula. El docente } \\
\text { detecta la necesidad } \\
\text { de aclarar conceptos a } \\
\text { toda el aula. }\end{array}$ & Proactiva & $\begin{array}{l}\text { - Vídeo } \\
\text { - Audio } \\
\text { - Capturas de pantalla } \\
\text { - Escrito }\end{array}$ \\
\hline $\begin{array}{l}\text { Preventivo. Previo a la } \\
\text { entrega de la PEC }\end{array}$ & $\begin{array}{l}\text { Identificación y } \\
\text { corrección de errores }\end{array}$ & $\begin{array}{l}\text { Grupo específico o in- } \\
\text { dividual. Los estudian- } \\
\text { tes plantean dudas y el } \\
\text { docente responde indivi- } \\
\text { dualmente o haciendo } \\
\text { agrupaciones. }\end{array}$ & Reactiva o proactiva & $\begin{array}{l}\text { - Escrito (reactivo) } \\
\text { - Repositorio multimedia } \\
\text { (proactivo) }\end{array}$ \\
\hline $\begin{array}{l}\text { Inmediato. Después de } \\
\text { la entrega de la PEC }\end{array}$ & Respuesta correcta & Aula. & Reactiva & $\begin{array}{l}\text { - Solución escrita de } \\
\text { las PEC }\end{array}$ \\
\hline $\begin{array}{l}\text { Diferido. Después de la } \\
\text { entrega de la PEC }\end{array}$ & Mejora de tareas & $\begin{array}{l}\text { Aula o grupo especí- } \\
\text { fico. El docente detecta } \\
\text { errores generalizados a } \\
\text { las respuestas y hace un } \\
\text { feedback para mejorar } \\
\text { futuras PEC. }\end{array}$ & Proactiva & $\begin{array}{l}\text { - Vídeo } \\
\text { - Audio } \\
\text { - Capturas de pantalla } \\
\text { - Escrito }\end{array}$ \\
\hline $\begin{array}{l}\text { Diferido. Después de la } \\
\text { entrega de la PEC }\end{array}$ & Mejora de tareas & $\begin{array}{l}\text { Individual. El docente } \\
\text { se dirige a los estudian- } \\
\text { tes que necesitan una } \\
\text { atención personalizada. }\end{array}$ & Proactiva & $\begin{array}{l}\text { - Vídeo } \\
\text { - Audio } \\
\text { - Capturas de pantalla } \\
\text { - Escrito }\end{array}$ \\
\hline
\end{tabular}

Fuente: Elaboración propia

\section{2. ¿Cómo podemos realizar un feedback sostenible?}

La elaboración de retornos individualizados por parte de los profesores consultores a las soluciones entregadas por los estudiantes, de cuatro o más actividades de evaluación continua, con aulas de 70 o más estudiantes, es a menudo una tarea insostenible por el tiempo que supone hacerlo. Hay que tener en cuenta que la dedicación estimada de los docentes colaboradores de la UOC es de un máximo de dos horas diarias. Necesitábamos, por lo tanto, encontrar herramientas y estrategias que permitiesen hacer retronos lo más personalizados posibles y, a la vez, no colapsar la tarea de los consultores.

Para dar respuesta a la pregunta inicial, desde los Estudios de Economía y Empresa de la UOC hemos realizado tres proyectos de innovación para explorar nuevas formas de feedback, con el objetivo de hacerlo más cercano, más eficiente y que genere motivación en el estudiante. Asimismo, atendiendo a la gran diversidad de profesores consultores que participan, diversidad entendida como las diferentes procedencias, profesiones y especialmente, las diferentes capacidades en la transmisión de los conocimientos, se ha investigado sobre las posibilidades de realizar feedbacks en formatos diversos que las tecnologías actuales ponen a nuestro alcance (audio, vídeo, captura de pantallas con audio, entre otros).

Los criterios metodológicos que se utilizaron en los tres pilotos fueron: (1) En la primera prueba piloto, se optó por hacer grupos de dimensiones reducidas para cada asignatura (máximo de 35 estudiantes), para asegurar la adecuada dimensión de la tarea del docente. En las pruebas piloto posteriores, los grupos fueron de dimensión normal; (2) se seleccionaron las asignaturas en función del tipo de conocimiento y metodología diversa, (3) se seleccionaron docentes con aptitudes diferentes, para valorar la transversalidad de la actuación y su posible extensión al conjunto de las asignaturas del grado, (4) después de cada prueba piloto, se ha realizado una encuesta a los estudiantes para saber cuál ha sido su percepción y utilidad del feedback recibido y, (5) los consultores que han participado también han contestado varios cuestionarios, han anotado sistemáticamente el tiempo empleado 
en la experiencia y han participado en diferentes focus groups presenciales para compartir las experiencias entre ellos.

Las asignaturas de las pruebas piloto requerían ser representativas de los diferentes ámbitos de conocimiento del grado. Desde la economía aplicada (Estructura económica y Comportamiento de los agregados económicos) a asignaturas más metodológicas (Fundamentos de estadística), pasando por asignaturas de contabilidad (Introducción a la contabilidad y Análisis de los estados financieros) y de organización de las empresas (Introducción a la empresa).

Los resultados obtenidos en las diferentes pruebas piloto muestran que para hacer un feedback sostenible se tienen que dar las siguientes condiciones:

En primer lugar, con el objetivo de hacer el feedback multimedia más eficiente y para aprovechar al máximo sus opciones, los docentes necesitan una formación específica, tanto tecnológica como pedagógica, así como de desarrollo de capacidades comunicativas mediante vídeo.

En segundo lugar, ha quedado comprobado que un feedback personalizado requiere una importante inversión de tiempo por parte del docente. Por lo tanto, conviene planificar la intensidad y la frecuencia con que se llevará a cabo el retorno y cambiar la forma de corregir la pruebas de evaluación continua.

En tercer lugar, la efectividad del feedback y la idoneidad de usar un canal u otro y un tipo u otro de feedback depende de la tipología de la asignatura y de las habilidades comunicativas y técnicas de los docentes.

Finalmente, podemos planificar dos estrategias de feedback: (1) para llegar a todos a los estudiantes que necesiten un feedback más personal, hay que poderlos discriminar y saber adaptar el mensaje que debe recibir cada cual, y (2) elaborar un repositorio de vídeos, audios o capturas de pantallas comentadas que nos permitan avanzarnos a las dudas que tradicionalmente salen en cada semestre.

\section{Una posible solución}

A continuación, en la tabla 2, proponemos una estrategia para elaborar un feedaback el máximo de individualizado posible, a la vez que compatible con la tarea de docente. Sugerimos llevar a cabo el feedback estableciendo cuatro categorías de estudiante.

Tabla 2. Modalidades de feedback

\begin{tabular}{|c|c|c|c|}
\hline Calificación & Contenido del feedback & A quién lo dirigimos & Nivel de personalización \\
\hline A & Mensaje de ánimo y felicitación & $\begin{array}{l}\text { Individual. El cuerpo del mensaje } \\
\text { puede ser parecido, pero hay que } \\
\text { personalizarlo resaltando aquello que } \\
\text { es más excelente. }\end{array}$ & Nivel de personalización bajo \\
\hline B & $\begin{array}{l}\text { Mensaje de ánimo y de felicitación + } \\
\text { comentar qué errores han cometido }\end{array}$ & $\begin{array}{l}\text { Grupo específico o individual. } \\
\text { Puede hacerse algún mensaje a to- } \\
\text { dos los estudiantes que han obtenido } \\
\text { una misma calificación o de forma } \\
\text { individual si la situación lo requiere } \\
\text { (especialmente C+) }\end{array}$ & Nivel de personalización medio \\
\hline $\mathrm{C}+, \mathrm{C}-$ y D & $\begin{array}{l}\text { Mensaje personal de ánimo, comen- } \\
\text { tando los errores más relevantes y } \\
\text { explicando alternativas para mejorar } \\
\text { el nivel de futuros ejercicios }\end{array}$ & $\begin{array}{l}\text { Individual. Es el más personalizado } \\
\text { de todos, hay que dar una respuesta } \\
\text { personal basada en la prueba entre- } \\
\text { gada por el estudiante. }\end{array}$ & Nivel de personalización alto \\
\hline N & $\begin{array}{l}\text { Recordatorio de que están a tiempo } \\
\text { de seguir la evaluación continua }\end{array}$ & $\begin{array}{l}\text { Individual. El cuerpo del mensaje } \\
\text { puede ser parecido, pero hay que } \\
\text { personalizarlo. }\end{array}$ & Nivel de personalización bajo \\
\hline
\end{tabular}

Fuente: Elaboración propia 


\section{4. ¿Es realmente efectivo el feedback individualizado?}

Los resultados obtenidos muestran que los estudiantes valoran muy positivamente el feedback personal, además de motivarlos a seguir con la asignatura en caso de obtener una baja calificación o no presentar un ejercicio. Concretamente, de la experiencia queremos destacar las cuatro ideas más relevantes que han manifestado los estudiantes sobre la importancia y necesidad que le dan al feedback.

En general, los estudiantes le dan más importancia al feedback en una enseñanza en línea que en una enseñanza presencial. Asimismo, nunca consideran excesivo o inadecuado el feedback recibido.

En segundo lugar, los estudiantes dan más importancia a la calidad del feedback y al grado de personalización que no a la cantidad, la tecnología utilizada o el momento de recibirlo. En este sentido, los estudiantes se muestran muy pragmáticos en el sentido de que valoran, por encima de todo, la utilidad del feedback recibido, mucho más que no el tipo de feedback o el canal (audio, vídeo, etc.) utilizado.

En tercer lugar, destaca que el factor más importante es el hecho de recibir feedback por parte del consultor. La comunicación interactiva con el docente es un elemento de motivación de primer nivel para los estudiantes. Concretamente, valoran especialmente que los mensajes sean claros y que se concrete mucho aquello que no han hecho bien y por qué motivo.

Finalmente, el estudiante acostumbra a adaptarse a las condiciones en que se trabaja en cada asignatura. Esto hace que, a pesar de las condiciones y herramientas sean mejorables, a menudo no se plantea ni reclama otras opciones o recursos. Ahora bien, una vez descubre (o se le muestra) que puede haber otras herramientas, como pueden ser vídeos, mensajes personalizados, correcciones particulares, etc., se acostumbra y lo reclama cuando no lo encuentra.

\section{Conclusiones}

La adaptación al Espacio Europeo de Enseñanza Superior, la necesidad de mejorar el proceso de aprendizaje y la motivación de los estudiantes -dando un tratamiento diferenciado a su propia diversidad- y, en definitiva, la necesidad de aumentar su satisfacción, implica que la realización de retornos personalizados de calidad sea un factor clave para continuar garantizando la calidad del modelo pedagógico de la UOC.

Hemos visto que los estudiantes valoran muy positivamente el feedback personalizado y que este tiene un efecto facilitador del aprendizaje, así como de motivación. Ahora bien, de acuerdo con las opiniones expresadas por los estudiantes, parece que valoran más el contenido de la comunicación que no el medio a través del cual se le transmite este mensaje. Podemos afirmar que el feedback personalizado acerca la relación entre los docentes y los estudiantes, y que tiene efectos que favorecen el aprendizaje y motivan al estudiante. También es cierto que, por el contrario, esta relación más personalizada entre docente y estudiante va en detrimento de la relación grupo aula.

También se ha puesto de manifiesto que la personalización del feedback cambia la manera de corregir las pruebas, y la utilización de otras maneras de hacer feedback, diferentes de la textual, no reduce la dedicación de los docentes y hace difícil su implantación generalizada en grupos de 70 estudiantes, sobre todo teniendo en cuenta que a medida que se hacen estos feedbacks individuales, los estudiantes se vuelven más exigentes y aumenta la interacción docente-estudiante.

A pesar de haber integrado estos comentarios en el marco del registro de evaluación continua (RAC) para minimizar el coste para el consultor, así como haber incorporado las herramientas para facilitar la adopción de distintas respuestas multimedia (grabaciones en vídeo, grabaciones de audio y la posibilidad de colgar vídeos comprimidos), se hace imprescindible continuar avanzando para asegurar la sostenibilidad de un retorno personalizado, estableciendo alguna estrategia de feedback concreta, como la que se hace en este artículo.

Finalmente, cabe destacar que para los estudiantes el elemento más importante del feedback es el contenido y no el medio a través del cual se transmite. Aun así, para que el feedback sea realizado por los docentes, hay que establecer canales para ofrecer a los consultores formación técnica, pegagócica y en comunicación . 


\section{Bibliografía}

ALLAL, L. K. (1979). "Stratégies d'évaluation formative: conceptions psychopédagogiques et modalités d'application». En: L. K. Allal, J. Cardinet, P. Perrenoud (eds.). L'evalutaion formative dans un enseignement différéncié. (6 $6^{a}$. ed., págs. 153-185). Institut Romand de recherches et de documentation pedagogiques.

ALLAL, L. K. (1988). «Vers un élargissement de la pédagogie de maîtrisse: Processus de régulation interactive, rétroactive et proactive». En: A. M. Humberman (ed.). Assurer la réussite des apprentissages scolaires? Les propositions de la pédagogie de maîtrisse (págs. 86-126). Neuchâtel: Delachaux and Niestlé.

BLACK, P.; WILIAM, D. (1998). "Assessment and Classroom Learning». Assessment in Education: Principles, Policy \& Practice. n. ${ }^{\circ}$ 5, vol. 1, págs. 7-74. <doi:10.1080/0969595980050102>

ESPASA, A. (2008). El Feedback en el marc de la regulació de l'aprenentatge: caracterització $i$ anàlisi en un entorn formatiu en línia. Universitat Oberta de Catalunya [tesis doctoral en línea]. <http://www.tdx.cat/browse?value $=$ Espasa+Roca\%2C+Anna\&type=author $>$

HATTIE, J. A. (1987). «ldentifying the Salient Facets of a Model of Student Learning: A Synthesis of Meta Analyses». International Journal of Educational Research, n. ${ }^{\circ} 11$, vol. 2, págs. 187-212.

HIGGINS, R.; HARTLEY, P.; \& SKELTON, A. (2002). «The Conscientious Consumer: Reconsidering the role of assessment feedback in student learning". Studies in Higher Education, n. ${ }^{\circ}$ 27, vol. 1, págs. 53-64. $<$ doi:10.1080/03075070120099368>

LAURILLARD, D. (2002). Rethinking university teaching: a conversational framework for the effective use of learning technologies. Routledge.

LEA, S. J.; STEPHENSON, D.; TROY, J. (2003). «Higher Education Students' Attitudes to Student-centred Learning: Beyond "educational bulimia"?». Studies in Higher Education, n. ${ }^{\circ} 28$, vol. 3, págs. 321-334. $<$ doi:10.1080/03075070309293>

MARTíNEZ-ARGÜELLES, M. J.: BADIA-MIRÓ, M.; HINTZMANN, C. [et al.] (2011). Personalized E-Feedback and information and communication technologies (ICT). Presentado en Edulearn11. Tercera Conferencia Internacional sobre Educación y Nuevas Tecnologías del Aprendizaje, celebrada en Barcelona. <http://www.iated. org/edulearn11/>

MARTÍNEZ-ARGÜELLES, M. J.; BATALLA, J. M.; NOGUERA, P. [et al.] (2011). Evaluation of Multimedia Tools and e-Feedback in Virtual Learning Environments. Presentado en Edulearn11. Tercera Conferencia Internacional sobre Educación y Nuevas Tecnologías del Aprendizaje, celebrada en Barcelona. <http://www.iated.org/edulearn11/>

NARCISS, S. (2004). «The Impact of Informative Tutoring Feedback and Self-Efficacy on Motivation and Achievement in Concept Learning". Experimental Psychology (formerly "Zeitschrift für Experimentelle Psychologie"), n. 51, vol. 3, págs. 214-228. <doi:10.1027/1618-3169.51.3.214>

PÉREZ CAÑADO, M. L. (2009). «Debunking EHEA Myths: Common ECTS Misconceptions and Why They Are Wrong». International Education Studies, n. ${ }^{\circ}$ 2, vol. 4, pág. 15. <doi:10.5539/ies.v2n4P15>

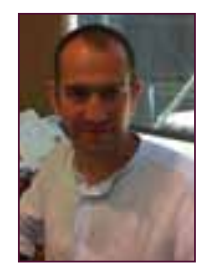

Josep-Maria Batalla-Busquets

jbatalla@uoc.edu

\section{Profesor de los Estudios de Economía y Empresa (UOC)}

Licenciado en Ciencias Económicas y Empresariales por la Universitat de Barcelona, doctor en Economía por la misma universidad. Profesor agregado de los Estudios de

Economía y Empresa de la UOC. Desde el 2012 coordina el grupo de investigación Management \& e-learning (MEL) adscrito al eLearn Center. Ha dirigido y participado en varios proyectos de innovación docente financiados por la UOC y la Agencia de Gestión de Ayudas Universitarias y de Investigación de la Generalitat de Catalunya.

$<$ http://www.uoc.edu/webs/jbatalla/ES/curriculum/index.html> 




\section{María-Jesús Martínez-Argüelles} mmartinezarg@uoc.edu

Licenciada en Ciencias Económicas y Empresariales por la Universidad de Oviedo. Doctora en Administración de Empresas por la Universitat de Barcelona. Profesora agregada de los Estudios de Economía y Empresa de la Universitat Oberta de Catalunya (UOC), donde dirige el grado de Administración y Dirección de Empresas. Su campo de especialización es el de organización de empresas. Es miembro del grupo de investigación Management \& eLearning (MeL).



\section{Dolors Plana Erta}

dplana@uoc.edu

Profesora de los Estudios de Economía y Empresa (UOC)

Profesora del área de contabilidad a los Estudios de Economía y Empresa de la UOC. Licenciada en Ciencias económicas y empresariales por la Universidad Autónoma de Barcelona y actualmente realizando la tesis doctoral en el programa de doctorado de Administración y dirección de empresas de la Universidad Politécnica de Catalunya. Ha participado en varios proyectos de innovación docente financiados por la UOC y la Agencia de Gestión de Ayudas Universitarias y de Investigación de la Generalitat de Catalunya $<$ http://www.uoc.edu/webs/dplana/ES/curriculum/index.html>

Los textos publicados en esta revista están sujetas -salvo que se indique el contrario- a una licencia de Reconocimiento 3.0 España de Creative Commons. Podéis copiarlos, distribuirlos, comunicarlos públicamente y hacer obras derivadas siempre que reconozcáis los créditos de las obras (autoría, nombre de la revista, institución editora) de la manera especificada por los autores o por la revista. La licencia completa se puede consultar a http://creativecommons.org/licenses/by/3.0/es/deed.ca .

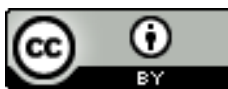

